\title{
Traffic Control Models Based on Cellular Automata for At-Grade Intersections in Autonomous Vehicle Environment
}

\author{
Wei Wu, ${ }^{1}$ Yang Liu, ${ }^{1}$ Yue Xu, ${ }^{2}$ Quanlun Wei, ${ }^{3}$ and Yi Zhang ${ }^{4,5}$ \\ ${ }^{1}$ School of Traffic and Transportation Engineering, Changsha University of Science and Technology, Changsha 410076, China \\ ${ }^{2}$ Polytech Nantes, Université de Nantes, 44600 Nantes, France \\ ${ }^{3}$ College of Transport and Communications, Shanghai Maritime University, Shanghai 201306, China \\ ${ }^{4}$ China Institute of Urban Governance, Shanghai Jiao Tong University, Shanghai 200240, China \\ ${ }^{5}$ School of Naval Architecture, Ocean and Civil Engineering, Shanghai Jiao Tong University, Shanghai 200240, China
}

Correspondence should be addressed to Yi Zhang; darrenzhy@sjtu.edu.cn

Received 29 July 2017; Revised 1 October 2017; Accepted 11 October 2017; Published 6 November 2017

Academic Editor: Xiaolei Ma

Copyright (C) 2017 Wei Wu et al. This is an open access article distributed under the Creative Commons Attribution License, which permits unrestricted use, distribution, and reproduction in any medium, provided the original work is properly cited.

\begin{abstract}
Autonomous vehicle is able to facilitate road safety and traffic efficiency and has become a promising trend of future development. With a focus on highways, existing literatures studied the feasibility of autonomous vehicle in continuous traffic flows and the controllability of cooperative driving. However, rare efforts have been made to investigate the traffic control strategies in autonomous vehicle environment on urban roads, especially in urban intersections. In autonomous vehicle environment, it is possible to achieve cooperative driving with V2V and V2I wireless communication. Without signal control, conflicted traffic flows could pass intersections through mutual cooperative, which is a remarkable improvement to existing traffic control methods. This paper established a cellular automata model with greedy algorithm for the traffic control of intersections in autonomous vehicle environment, with autonomous vehicle platoon as the optimization object. NetLogo multiagent simulation platform model was employed to simulate the proposed model. The simulation results are compared with the traffic control programs in conventional Synchro optimization. The findings suggest that, on the premises of ensuring traffic safety, the control strategy of the proposed model significantly reduces average delays and number of stops as well as increasing traffic capacity.
\end{abstract}

\section{Introduction}

The emergence and development of motorized transportation have not only promoted the progress of human civilization, but also brought about severe issues relating to road safety and traffic efficiency. According to statistics, more than 100,000 people died in road accidents per year worldwide [13]. In the United States traffic congestion has caused enormous economic losses. For example, in 2013, urban Americans experienced an extra 6.8 billion hours of travel and 3.1 billion gallons of fuel consumed because of traffic congestion [4-6]. With the development of motor vehicle, researchers have recognized that automata vehicle is probably one of the most effective ways to tackle those problems [7-10].

In the autonomous vehicle environment, real-time, mutual, and effective communication could be achieved not only between vehicles and vehicles, but also between vehicles and road [11-14]. With respect to road safety, the mutual cooperation helps to solve the traffic problems caused by the driving errors, insufficient visibility distance [15-17]. In terms of traffic efficiency, vehicles could form platoon through cooperation to reduce headways and increase road capacity [18-20].

A major part of previous studies on traffic flow in autonomous vehicle environment focused on continuous flow and the cooperative between vehicles on highway [21-23]. Few studies have proposed traffic control strategies in autonomous vehicle environment, with an emphasis on intermittent traffic flows. Considering the limitation of road space, even in autonomous vehicle environment, there will still be large number of plane intersections in urban areas. Therefore, it is imperative to investigate the traffic control strategies in autonomous vehicle environment in plane intersections. Although some related researches proposed traffic control 


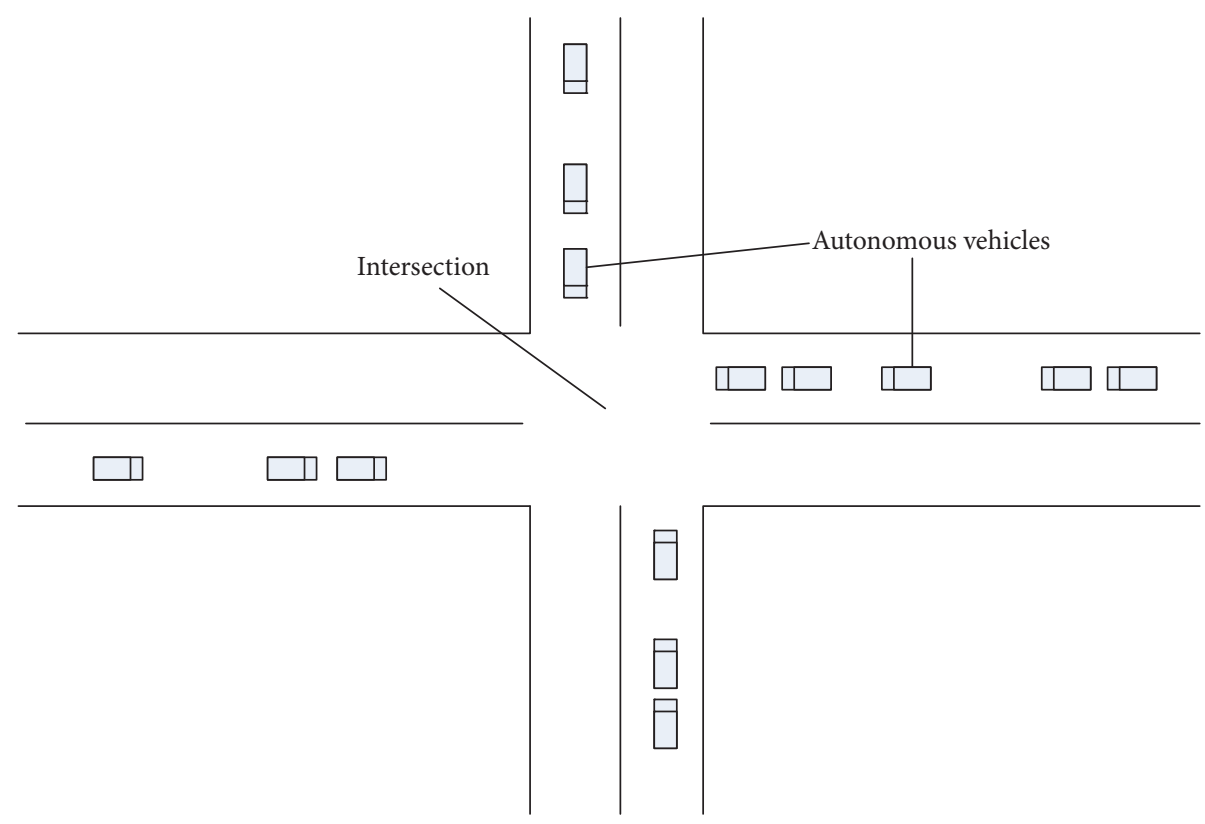

FIgURE 1: Basic idea of the research problem.

model in autonomous vehicle environment, the control algorithm is mainly on the basis of first-come-first-served (FCFS) algorithm [24], and the optimized object is focused on every single vehicle $[25,26]$ and thus has limited optimization effects.

Focusing on a single intersection, this paper proposed a traffic control model based on cellular automata in autonomous vehicle environment. The primary goals of this paper are to (1) explicitly capture the dynamic interaction between platoon and traffic control strategy; (2) establish cellular automata model to interpret the disciplines of traffic flow in autonomous vehicle environment; and (3) improve traffic efficiency based on minimize travel delays.

\section{Development of Optimization and Simulation Approach}

2.1. Problem Description. In autonomous vehicle environment, traffic lights are unnecessary. Traffics travel through plane intersections by communicating with each other. The research problem can be demonstrated in Figure 1. Autonomous vehicles arrive stochastically from four approaches. For the sake of calculation simplicity, in this paper, there is only one through lane considered at each approach. Pedestrians and cyclists are not considered. How to allocate passage time for each autonomous vehicle to maximize the system benefits of the intersection is studied in this paper. The common solution to this problem is first-come-first-served (FCFS) algorithm. However, FCFS algorithm has some disadvantages that can be demonstrated in Figure 2. There are two southbound autonomous vehicles in Figure 2. The estimated arrival times for these two vehicles are 2 and 5 seconds, respectively. There are a bunch of continuous westbound autonomous vehicles. The estimated arrival times are 3, 3.5,
4, 4.5, 5, 5.5, and 6 seconds, respectively. By employing FCFS algorithm, the southbound autonomous vehicle with 2 seconds' estimated arrival time will be served firstly. However, maybe better solution can be obtained if the continuous vehicle platoons can be able to proceed with priority. On the other hand, the optimization algorithm on account of each vehicle is very complex and inefficient. Optimization based on vehicle platoons may have applicability.

2.2. General Notation and Terminology. The notations used hereafter are summarized in Key Variables (Notations) Used in the Formulations.

2.3. The Traffic Flow Model Based on the Cellular Automata. Discrete time and discrete space are employed in cellular automata model. It is widely used in modeling traffic flows as it is able to simulate complicated traffic flow phenomena with a small number of simple rules. The basic traffic flow models based on cellular automata are as follows, from vehicle 1 to vehicle $n$.

The Acceleration Process. When an autonomous vehicle proceeds freely, it accelerates to the maximum speed, which is presented by the following formula.

$$
\begin{gathered}
\text { If }\left(V_{i}^{j}(t)<\Delta X_{i-1}^{j}(t)+G_{i}^{j}(t)\right): \\
\left\{I f\left(V_{i}^{j}(t)<V_{\max }\right),\right. \\
V_{i}^{j}(t+1)=V_{i}^{j}(t)+1
\end{gathered}
$$

else

$$
\left.V_{i}^{j}(t+1)=V_{\max }\right\}
$$




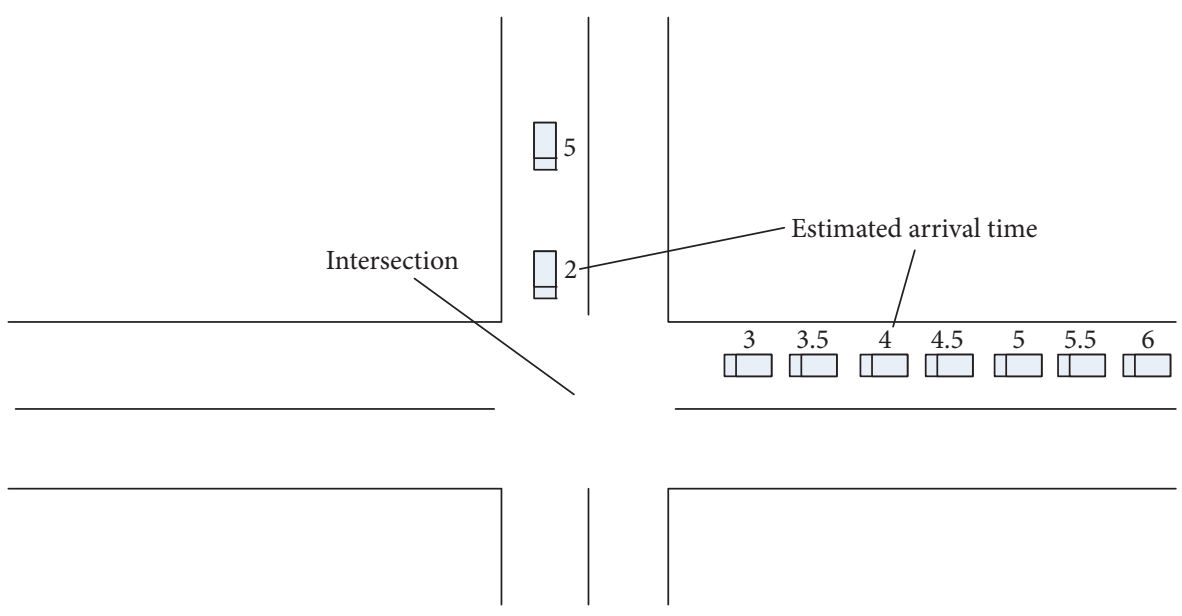

FIGURE 2: Disadvantages of first-come-first-served (FCFS) algorithm.

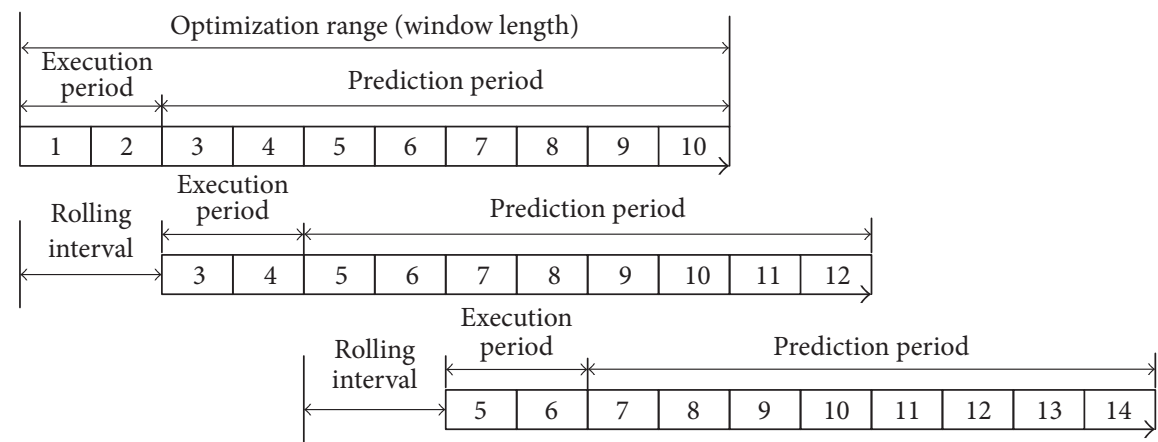

FIGURE 3: Optimization method of sliding time window.

The Deceleration Process. When an autonomous vehicle is blocked by a leading vehicle, it decelerates to avoid a crash and closely follows the leading vehicle.

$$
\text { If } \quad \begin{aligned}
\left(V_{i}^{j}(t) \geq \Delta X_{i-1(t)}^{j}+G_{i}^{j}(t)\right): \\
\quad\left\{V_{i}^{j}(t+1)=\min \left(\Delta X_{i-1}^{j}(t)+G_{i}^{j}(t), V_{i}^{j}(t)\right)\right\} .
\end{aligned}
$$

When an autonomous vehicle arrives at the intersection and does not get right-of-way, it decelerates as follows:

$$
\text { If } \begin{aligned}
\left(V_{i}^{j}(t) \geq D_{i}^{j}(t)\right): \\
\left\{I f\left(\sigma_{i}^{j}(t)=1\right),\right. \\
V_{i}^{j}(t+1)=\min \left(\Delta X_{i-1}^{j}(t)+G_{i}^{j}(t), V_{i}^{j}(t)\right) \\
\text { If }\left(\sigma_{i}^{j}(t)=0\right), \\
\left.V_{i}^{j}(t+1)=\min \left(D_{i}^{j}(t), \Delta X_{i-1}^{j}(t)+G_{i}^{j}(t), V_{i}^{j}(t)\right)\right\} .
\end{aligned}
$$

The Platoon Merging Process. If the time headway between two vehicles at the same approach is less than the critical time headway, then the two vehicles will be treated as platoons.

$$
\text { If }\left(\left(X_{n}^{j-1}(t)-X_{1}^{j}(t)\right) / V_{1}^{j}(t)<h_{c}\right):
$$

\{merging platoon $j$ into platoon $j-1$ \}.
The Platoon Split Process. When the waiting time of the traffics at the conflicting approach exceeds the maximum limit, the current vehicle platoon splits to two platoons, so as to give right-of-way to the conflicting traffic flows.

\section{The Location Updating Process}

$$
X_{i}^{j}(t+1)=X_{i}^{j}(t)+V_{i}^{j}(t+1) .
$$

2.4. Rolling Window Time Control Strategy Based on Greedy Algorithm. In this paper, we employed the rolling time window approach as the optimization method. The basic process of the rolling time window approach is shown in Figure 3.

In the rolling step, we employed the greedy algorithm to optimize the control strategy of the platoon at the intersection. The optimization steps are as follows.

Step 1 (the beginning of optimization process). Identify the vehicle platoon on the four approaches. Optimization is started based on the platoon.

Step 2 (the determination of analysis scope). In the time span of each rolling step, search and determine the number of platoons that can pass through the four approaches.

Step 3 (the determination of control strategy). We number the four platoons that are closest to the intersections in 
the four approaches of analysis scope, assuming that the length of each platoon is $h, k, p, q$. Using the greedy algorithm, when the 4 platoons request the conflicting rightof-way, we selected the longest platoon $(\max (h, k, p, q))$ to pass through the intersection. When a platoon has already passed, we reselect another four platoons that are closest to the intersections in the four approaches and return to the beginning of Step 3. The maximal waiting time is set for each platoon. When the actual waiting time exceeds the maximal waiting time, the platoon has the highest priority.

Step 4. Go to the next rolling time and begin from Step 1.

\section{Simulation Modeling}

In this paper, the simulation of traffic control model of plane intersection based on cellular automata in automatic driving environment is conducted in NetLogo platform. NetLogo is a multiagent programmable modeling environment for simulating natural and social phenomena. It was launched by UriWilensy in 1999 and was developed by the Link Learning and Computer Modeling Center (CCL), which is designed to provide a powerful and easy-to-use computer-aided tool for research and educational institutions.

At numerical simulation, each cellular length takes $3.5 \mathrm{~m}$. $V_{\max }=6(76 \mathrm{~km} / \mathrm{h})$; the simulation step size was set to 0.1 seconds, with continuous operation of 36000 steps of onehour traffic conditions, taking into account the temporal and spatial distribution of traffic flow characteristics, using open boundary conditions. In the model, in order to eliminate the impact of random factors, using the last 900 simulation steps (15 minutes) for sample calculation, every sample represents 20 simulations, that is, each point of the chart is the average of 20 simulations.

\section{Performance Analysis}

To evaluate the performance of the proposed strategies, we compared the control strategy of proposed model and the conventional signal control strategy optimized by Synchro software. In Synchro model, the saturation flow was set to $1900 \mathrm{pcu} / \mathrm{h}$; the amber time and all red time were set to $3 \mathrm{~s}$ and $2 \mathrm{~s}$, respectively. Green splits and green phase time are optimized by Synchro software automatically. By adjusting the amount of approaching traffic volume, we changed the degree of saturation in the range of 0.1 to 1.3 (based on the saturation in Synchro). The length of the approach lane was set to $1000 \mathrm{~m}$. The maximum waiting time was set to $30 \mathrm{~s}$. The critical platoon headway was set to $2 \mathrm{~s}$. The results are shown in Figures 4 and 5.

As we can see from Figures 4 and 5, in both proposed model and Synchro model, the delays and number of stops increase with the increase of saturation. In Synchro model, different cycle time shows similar effects. When the saturation is below 0.8 , the delays and stops increase slowly. When the saturation is greater 0.8 , the delays and stops increase significantly. Compared with Synchro model, in the proposed model, when the saturation is high, the delays and stops will be much lower in autonomous vehicle environment. When

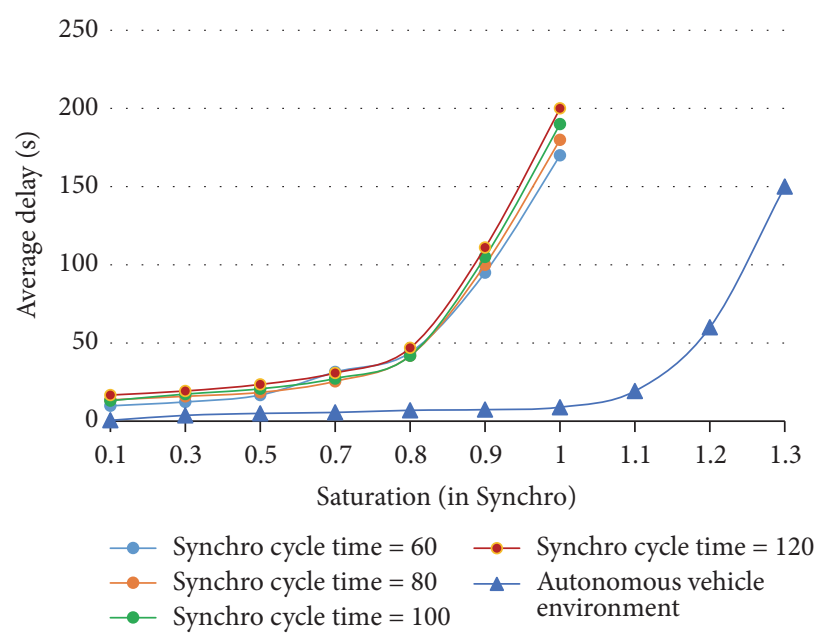

Figure 4: Comparison of average delay.

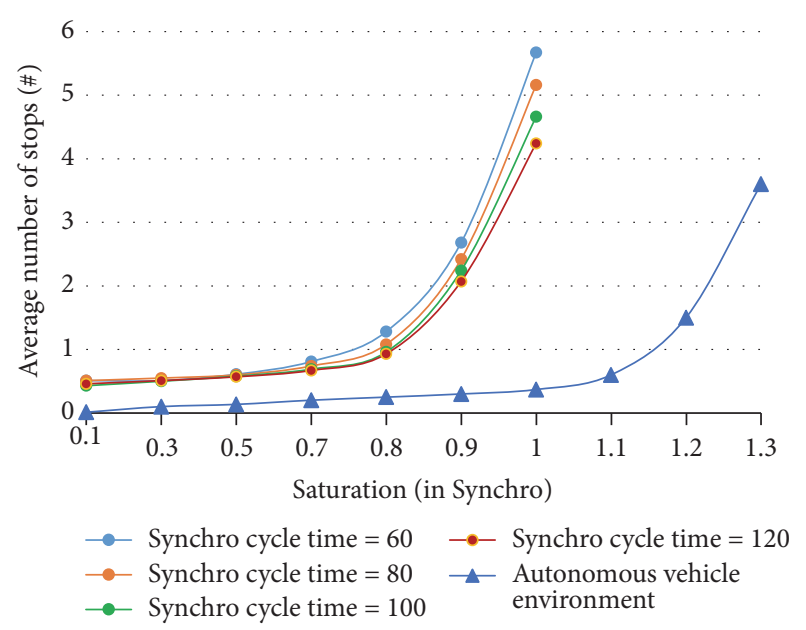

FIGURE 5: Comparison of average stops.

the saturation is as high as 1.1, the delays and stops will show significant rise in the proposed model. The findings represent that the proposed model is effective in reducing delays and stops.

As we can see from Figure 6, when the saturation is less than 0.5 , the numbers of vehicles clearing the intersection in the proposed model and Synchro model are almost the same, indicating that both models meet the needs. However, as the saturation continues to increase, the numbers of vehicles clearing the intersection in the proposed model and Synchro model will show significant differences. When the saturation is close to 0.9 , the number of vehicles clearing the intersection in Synchro model reaches maximum value. Meanwhile, when the saturation continues to rise to 1.1, the number of vehicles clearing the intersection in the proposed model is approaching stability. In the proposed model, the number of vehicles clearing the intersection when stable is $53.3 \%$ bigger than in Synchro model, suggesting that the proposed model is able to significantly improve traffic capacity. 


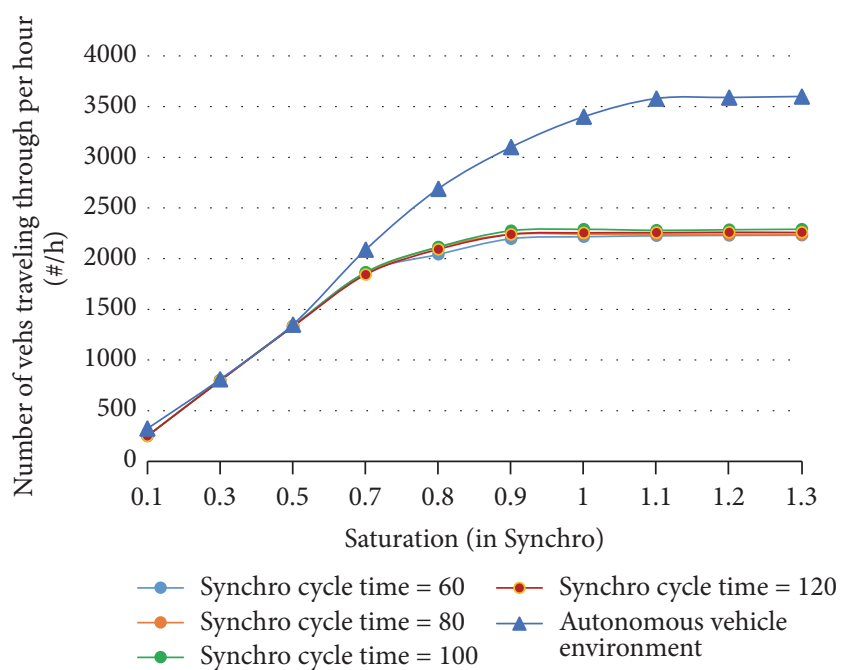

FIGURE 6: Comparison of number of vehicles passing per hour.

\section{Conclusions}

This paper presented a method of traffic control in the environment of automatic driving. Platoon is employed as the basic control unit. First, we establish the basic cellular automaton model. Then we optimized the control strategy based on rolling time window and greedy algorithm. Finally in this article, we employed the NetLogo multiagent simulation platform to simulate the proposed model. The simulation results show that, compared with Synchro model, the proposed model is able to reduce delays and the number of stops more effectively and to substantially increase the capacity of the intersection, which demonstrates the superiority of the proposed model.

This paper proposed preliminary simulation results and analysis for the proposed method at an isolated intersection. More extensive simulation experiments and field tests are required to be conducted in the future to assess the effectiveness of the proposed model under various traffic flow patterns. Corridor-wide evaluation of the proposed model's effectiveness should also be studied in future researches.

\section{Key Variables (Notations) Used in the Formulations}

\footnotetext{
$i$ : $\quad$ Vehicle $i$

$j: \quad$ Platoon $j$

$t$ : Time point

$V_{i}^{j}(t)$ : The speed of vehicle $i$ in platoon $j$ at time $t$ $(\mathrm{m} / \mathrm{s})$

$G_{i}^{j}(t)$ : The headway between vehicle $i$ in platoon $j$ and the vehicle in the front $(\mathrm{m})$

$D_{i}^{j}(t)$ : Distance from the vehicle $i$ in platoon $j$ to the intersection $(\mathrm{m})$

$\sigma_{i}^{j}(t)$ : Binary variable to represent if a vehicle gets right-of-way to travel through the intersection. If so, $\sigma_{i}^{j}(t)=1$. If not $\sigma_{i}^{j}(t)=0$
}

$T_{i}^{j}$ : The maximum acceptable waiting time at the intersection (s)

$\Delta X_{i}^{j}(t)$ : The moving distance of vehicle $i$ in platoon $j$ at time $t(\mathrm{~m})$

$V_{\max }: \quad$ The maximum speed of the vehicle $(\mathrm{m} / \mathrm{s})$

$n: \quad$ The maximum number of vehicles in the platoon (\#veh)

$h_{c}: \quad$ Critical time headway (s)

$\rho(t): \quad$ Average density at time $t(\mathrm{pcu} / \mathrm{km})$

$N(t)$ : Sum of the vehicles at time $t$ (\#veh).

\section{Conflicts of Interest}

The authors declare that there are no conflicts of interest regarding the publication of this paper.

\section{Acknowledgments}

The research is supported by the National Natural Science Foundation of China under Grant no. 61773077, no. 51408065, and no. 51308336, the Fundamental Research Funds for the Central Universities of Shanghai Jiao Tong University (Projects 15JCZZ05 and 16JXYB08), the Open Fund of the State Engineering Laboratory of Highway Maintenance Technology (Changsha University of Science \& Technology, Project KFJ160106), and the Urban China Initiative (UCI) Grant Project.

\section{References}

[1] H.-Y. Berg, J. Ifver, and M. Hasselberg, "Public health consequences of road traffic injuries - Estimation of seriously injured persons based on risk for permanent medical impairment," Transportation Research Part F: Traffic Psychology and Behaviour, vol. 38, pp. 1-6, 2016.

[2] Y. C. Huang, R. Cheng-Shyuan, C. Jung-Fang et al., "Characteristics and outcomes of patients injured in road traffic crashes and transported by emergency medical services," International Journal of Environmental Research \& Public Health, vol. 13, no. 2, article 236, 2016.

[3] C. Ding, D. Wang, C. Liu et al., "Exploring the influence of built environment on travel mode choice considering the mediating effects of car ownership and travel distance," Transportation Research Part A Policy \& Practice, vol. 100, pp. 65-80, 2017.

[4] D. Schrank, B. Eisele, and T. Lomax, "2014 Urban mobility report: powered by Inrix Traffic Data," Tech. Rep., Southwest Region University Transportation Center, Texas, Tex, USA, 2015.

[5] W. Wu, W. Ma, K. Long, H. Zhou, and Y. Zhang, "Designing sustainable public transportation: Integrated optimization of bus speed and holding time in a connected vehicle environment," Sustainability, vol. 8, no. 11, article 1170, 2016.

[6] C. Ding, C. Liu, Y. Zhang, J. W. Yang, and Y. P. Wang, "Investigating the impacts of built environment on vehicle miles traveled and energy consumption: differences between commuting and non-commuting trips," Cities, vol. 68, pp. 25-36, 2017.

[7] G. V. Raffo, G. K. Gomes, J. E. Normey-Rico, C. R. Kelber, and L. B. Becker, "A predictive controller for autonomous vehicle path tracking," IEEE Transactions on Intelligent Transportation Systems, vol. 10, no. 1, pp. 92-102, 2009. 
[8] N. Kehtarnavaz, N. Griswold, K. Miller, and P. Lescoe, "A transportable neural-network approach to autonomous vehicle following," IEEE Transactions on Vehicular Technology, vol. 47, no. 2, pp. 694-702, 1998.

[9] J. E. Naranjo, C. González, R. García, and T. De Pedro, "Lanechange fuzzy control in autonomous vehicles for the overtaking maneuver," IEEE Transactions on Intelligent Transportation Systems, vol. 9, no. 3, pp. 438-450, 2008.

[10] F. Jiménez, M. Clavijo, J. E. Naranjo, and Ó. Gómez, "Improving the Lane Reference Detection for Autonomous Road Vehicle Control," Journal of Sensors, vol. 2016, Article ID 9497524, 2016.

[11] K. Tsukamoto, Y. Oie, H. Kremo, O. Altintas, H. Tanaka, and T. Fujii, "Implementation and performance evaluation of distributed autonomous multi-hop vehicle-to-vehicle communications over TV white space," Mobile Networks and Applications, vol. 20, no. 2, pp. 203-219, 2015.

[12] L. Xu, L. Y. Wang, G. Yin, and H. Zhang, "Impact of communication erasure channels on the safety of highway vehicle platoons," IEEE Transactions on Intelligent Transportation Systems, vol. 16, no. 3, pp. 1456-1468, 2015.

[13] W. Wu, P. K. Li, and Y. Zhang, "Modelling and simulation of vehicle speed guidance in connected vehicle environment," International Journal of Simulation Modelling, vol. 14, no. 1, pp. 145-157, 2015.

[14] H. Peng, d. li, Q. Ye et al., "Resource allocation for cellular-based inter-vehicle communications in autonomous multiplatoons," IEEE Transactions on Vehicular Technology, vol. 99, pp. 1-15, 2017.

[15] F. Jiménez, J. E. Naranjo, and Ó. Gómez, “Autonomous collision avoidance system based on accurate knowledge of the vehicle surroundings," IET Intelligent Transport Systems, vol. 9, no. 1, pp. 105-117, 2015.

[16] P. Koopman and M. Wagner, "Autonomous vehicle safety: an interdisciplinary challenge," IEEE Intelligent Transportation Systems Magazine, vol. 9, no. 1, pp. 90-96, 2017.

[17] C. Wuthishuwong, A. Traechtler, and T. Bruns, "Safe trajectory planning for autonomous intersection management by using vehicle to infrastructure communication," EURASIP Journal on Wireless Communications and Networking, vol. 2015, no. 1, pp. 1-12, 2015.

[18] S. Gong, J. Shen, and L. Du, "Constrained optimization and distributed computation based car following control of a connected and autonomous vehicle platoon," Transportation Research Part B: Methodological, vol. 94, pp. 314-334, 2016.

[19] V. A. C. van den Berg and E. T. Verhoef, "Autonomous cars and dynamic bottleneck congestion: the effects on capacity, value of time and preference heterogeneity," Transportation Research Part B: Methodological, vol. 94, pp. 43-60, 2016.

[20] Y. Xie, H. Zhang, N. H. Gartner, and T. Arsava, "Collaborative merging strategy for freeway ramp operations in a connected and autonomous vehicles environment," Journal of Intelligent Transportation Systems: Technology, Planning, and Operations, vol. 21, no. 2, pp. 136-147, 2017.

[21] S. E. Shladover, "Cooperative (rather than autonomous) vehicle-highway automation systems," IEEE Intelligent Transportation Systems Magazine, vol. 1, no. 1, pp. 10-19, 2009.

[22] J. Hu, L. Kong, W. Shu, and M.-Y. Wu, "Scheduling of connected autonomous vehicles on highway lanes," in Proceedings of the 2012 IEEE Global Communications Conference (GLOBECOM '12), pp. 5556-5561, Anaheim, Calif, USA, December 2012.

[23] J. Wei, J. M. Dolan, and B. Litkouhi, "Autonomous vehicle social behavior for highway entrance ramp management," in
Proceedings of the IEEE Intelligent Vehicles Symposium (IV '13), pp. 201-207, Gold Coast, QLD, Australia, June 2013.

[24] K. Dresner and P. Stone, "A multiagent approach to autonomous intersection management," Journal of Artificial Intelligence Research, vol. 31, pp. 591-656, 2008.

[25] Z. Li, M. V. Chitturi, L. Yu, A. R. Bill, and D. A. Noyce, "Sustainability effects of next-generation intersection control for autonomous vehicles," Transport, vol. 30, no. 3, pp. 342-352, 2015.

[26] F. Zhu and S. V. Ukkusuri, "A linear programming formulation for autonomous intersection control within a dynamic traffic assignment and connected vehicle environment," Transportation Research Part C: Emerging Technologies, vol. 55, pp. 363378, 2015. 


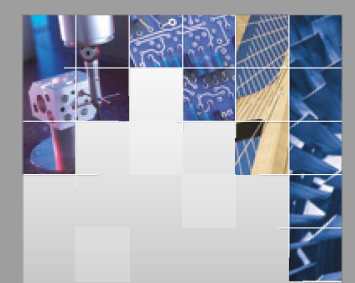

\section{Enfincering}
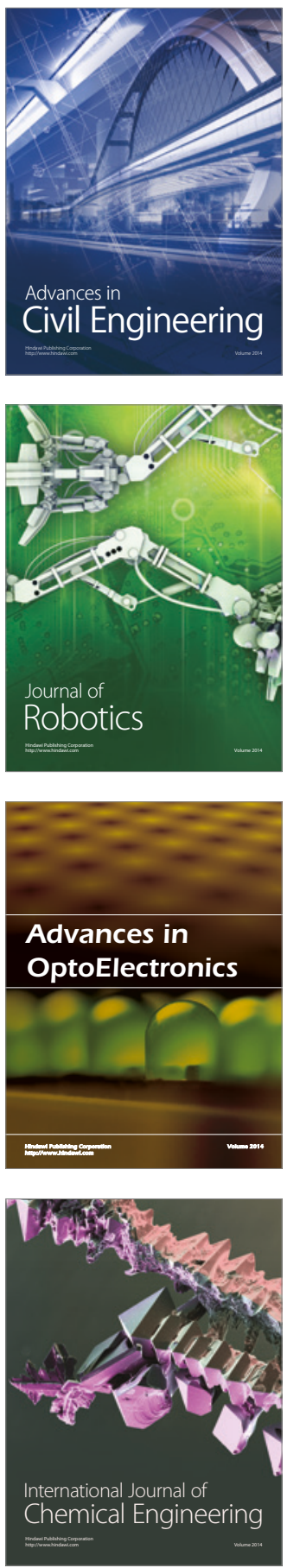

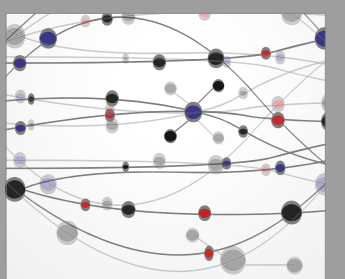

The Scientific World Journal

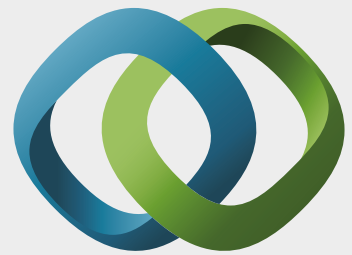

\section{Hindawi}

Submit your manuscripts at

https://www.hindawi.com
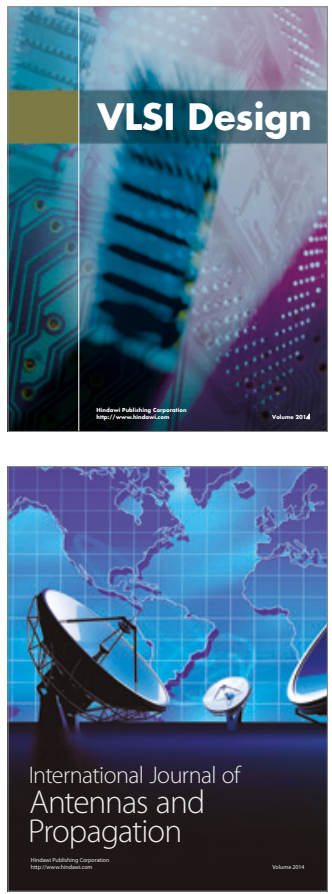

\section{Rotating}

Machinery
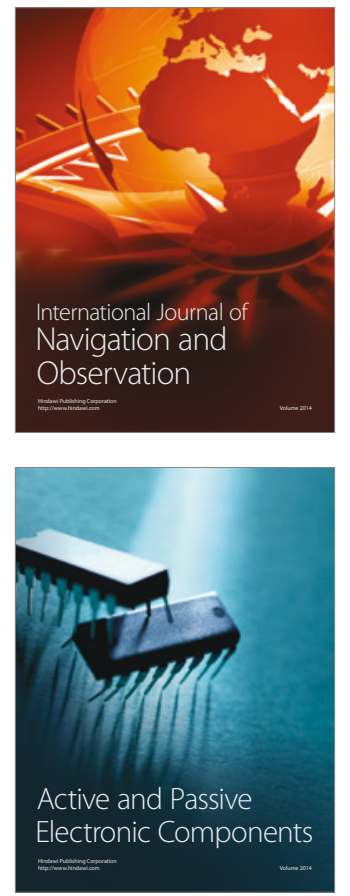
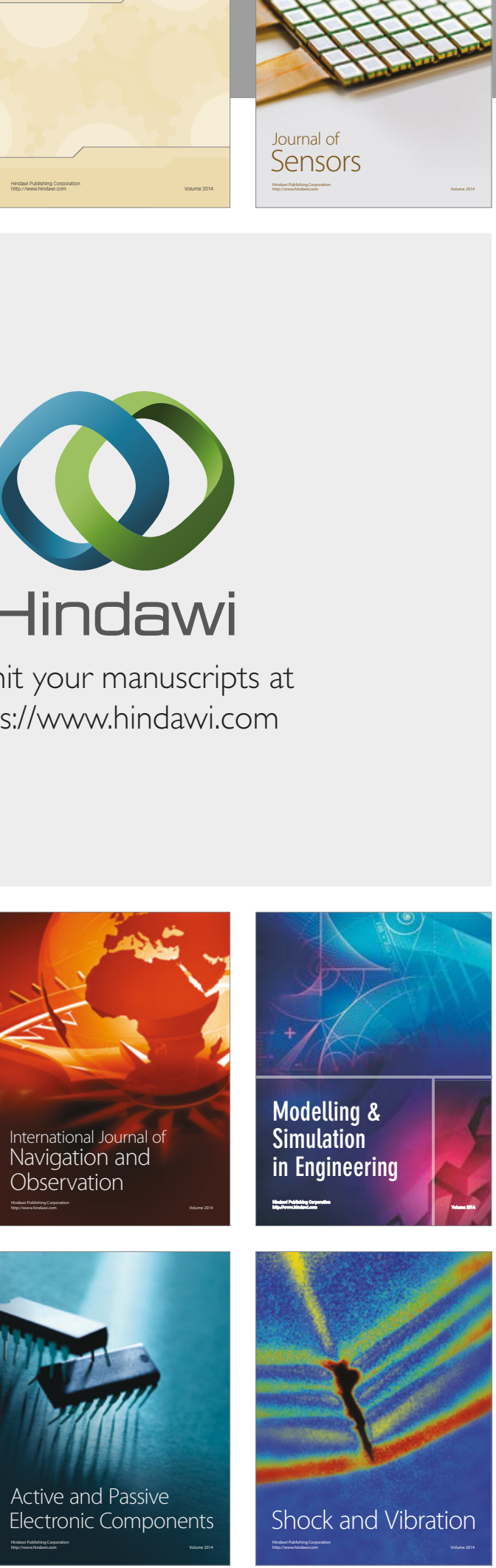
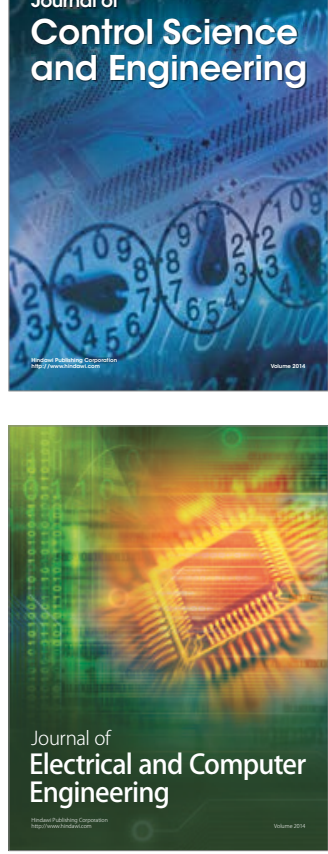

Distributed

Journal of

Control Science

and Engineering
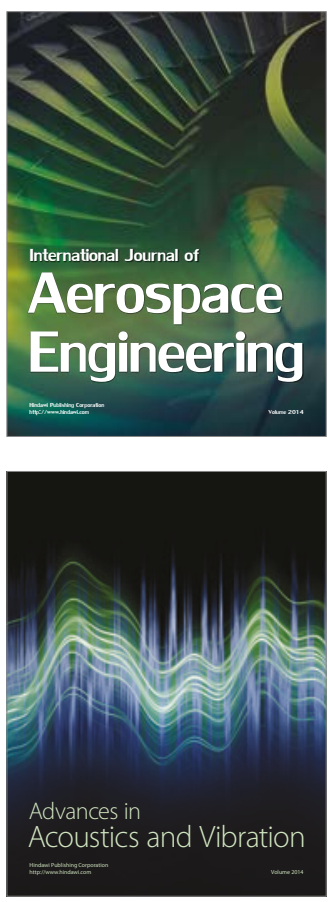

Sensor Networks 